\title{
TEORIAS CIENTÍFICAS DE SAÚDE NO CUIDADO AO PACIENTE ONCOLÓGICO: REVISÃO INTEGRATIVA
}

\section{SCIENTIFIC HEALTHCARE THEORIES IN ONCOLOGICAL PATIENT CARE: AN INTEGRATIVE REVIEW}

\section{TEORÍAS CIENTÍFICAS DE LA SALUd EN LA ATENCIÓN AL PACIENTE ONCOLÓGICO: UNA REVISIÓN INTEGRATIVA}

Isaura Letícia Tavares Palmeira Rolim', Lívia Maia Pascoal ${ }^{2}$, Adriana Gomes Nogueira Ferreira ${ }^{3}$, Janielle Ferreira de Brito Lima ${ }^{4}$, Luciana Léda Carvalho Lisbôa ${ }^{5}$, Thalita dos Santos Costa ${ }^{6}$

\section{RESUMO}

Objetivo: Analisar o uso das teorias de saúde no cuidado a pacientes oncológicos, destacando os principais eixos na assistência de enfermagem oncológica. Método: Trata-se de uma revisão integrativa da literatura realizada, por meio das bases de dados LILACS, PUBMED e MEDLINE, totalizando 16 publicações. Resultados: Após a análise, foi possível destacar 4 eixos principais na assistência de enfermagem oncológica: câncer, enfermagem e cuidado fundamentado em teorias; o autocuidado e o paciente oncológico; processo de ação, reação e interação na enfermagem; outras formas de cuidar. Conclusão: É necessário o avanço da ciência no campo da enfermagem oncológica e suas interações com as teorias científicas. $\mathrm{O}$ aprofundamento do conhecimento mostrará o caminho para um cuidado qualificado e orientado à oncologia.

Descritores: Teoria de enfermagem; Neoplasias; Cuidados de Enfermagem.

\begin{abstract}
Objective: To analyze the use of health theories in the care of cancer patients, highlighting the main axes in oncology nursing care. Method: This is an integrative literature review carried out using the LILACS, PUBMED and MEDLINE databases, totaling 16 publications. Results: After analysis, it was possible to highlight 4 main axes in oncology nursing care: cancer, nursing and care based on theories; self-care and cancer patients; action, reaction and interaction process in nursing; other ways of caring. Conclusion: It is necessary to advance science in the field of oncology nursing and its interactions with scientific theories. The deepening of knowledge will show the way to qualified care and oriented to oncology.
\end{abstract}

Descriptors: Nursing Theory; Neoplasms; Nursing Care.

\section{RESUMEN}

Objetivo: analizar el uso de las teorías de la salud en la atención de pacientes con cáncer, destacando los ejes principales en la atención de enfermería oncológica. Método: esta es una revisión de literatura integradora realizada utilizando las bases de datos LILACS, PUBMED y MEDLINE, con un total de 16 publicaciones. Resultados: después del análisis, fue posible destacar 4 ejes principales en la atención de enfermería oncológica: cáncer, enfermería y atención basada en teorías; autocuidado y pacientes con cáncer; proceso de acción, reacción e interacción en enfermería; otras formas de cuidar. Conclusión: es necesario avanzar en la ciencia en el campo de la enfermería oncológica y sus interacciones con las teorías científicas. La profundización del conocimiento mostrará el camino hacia la atención de calidad y orientada a la oncología.

Descriptores: Teoría de Enfermería; Neoplasias; Atención de Enfermería.

${ }^{1}$ Doutora em Enfermagem. Docente do Curso de Enfermagem da Universidade Federal do Maranhão; ${ }^{2}$ Doutora em Enfermagem. Docente do Departamento de Enfermagem da Universidade Federal do Maranhão; ${ }^{3}$ Doutora em Enfermagem. Docente do Departamento de Enfermagem da Universidade Federal do Maranhão; ${ }^{4}$ Doutoranda em Saúde Coletiva pela Universidade Federal do Maranhão. Enfermeira Assistencial do Hospital Universitário da UFMA; ${ }^{5}$ Mestre em Enfermagem. Enfermeira Supervisora das Clínicas Cirúrgicas do Hospital Aldenora Bello; ${ }^{6}$ Pós-graduanda em Enfermagem na Terapia Intensiva. Enfermeira Assistencial do Hospital do Servidor Estadual - HSLZ

Como citar este artigo:

Rolim, ILTP, Pascoal, LM, Ferreira, AGN, et al. Teorias científicas de saúde no cuidado ao paciente oncológico: revisão integrativa. Revista de Enfermagem do Centro-Oeste Mineiro. 2020;10:e3654. [Access http://doi.org/10.19175/recom.v10i0.3654 


\section{INTRODUÇÃO}

Câncer é o nome dado a uma série de doenças que têm em comum um descontrolado crescimento e divisão celular. Essas células anômalas, podem se multiplicar e invadir outros órgãos, em um processo conhecido por metástase. O câncer é um problema de saúde pública, ocupando o segundo lugar em mortalidade no mundo. No Brasil, com a mudança do perfil de morbimortalidade das últimas décadas e modificações socioculturais, houve crescimento no número de óbitos ligados a doenças crônicas ${ }^{(1)}$.

Há necessidade da atuação da enfermagem na educação, nos serviços de rastreamento da doença, nas pesquisas e investigações científicas, nas políticas de saúde e na criação de estratégias para prevenção e deteç̧ão precoce. Minimizando, dessa forma, os números de novos casos e de mortalidade por câncer e controlando o que temos hoje como um problema de saúde pública ${ }^{(2)}$.

A enfermagem evoluiu de práticas empíricas para uma ciência. A partir do século XIX, a Enfermagem Moderna ascende personificada pela figura de Florence Nightingale, que, em 1860, lançou os primeiros olhares voltados à enfermagem científica. Relacionava saúde às condições do ambiente, visando à colocação do doente em melhores situações para que a própria natureza promovesse a cura. As teorias de enfermagem são guias para as práticas de enfermagem, uma vez que oferecem um suporte científico para a sistematização da assistência de enfermagem, impactando no atendimento ao indivíduo com câncer, buscando manter ou melhorar a qualidade de vida ${ }^{(3)}$.

Aplicar fundamentos teóricos ao exercício assistencial da enfermagem permite 0 desenvolvimento de um cuidar sensível aos desejos, anseios, medos, potencialidades e necessidades do ser assistido. As teorias fornecem fundamentação científica necessária ao entendimento do enfermeiro frente aos valores ligados à sua prática assistencial e o real significado das respostas do indivíduo no seu processo saúde-doença( ${ }^{(3)}$, partindo dessa ideologia, justifica-se este estudo.

Com o passar dos anos, outros enfermeiros escreveram sobre a prática profissional, havendo grande busca no sentido de elaborar e desenvolver teorias e modelos conceituais de enfermagem A partir da década de 50 , com a tentativa de organizar o conhecimento pelos modelos conceituais de enfermagem, estabeleceram-se quatro conceitos ditos essenciais à prática profissional: o indivíduo, a saúde, o ambiente e a enfermagem ${ }^{(4)}$.

Ao se considerar o câncer como um problema de saúde pública atual e a complexidade das atividades de enfermagem inerentes aos cuidados do indivíduo com câncer, objetiva-se analisar o uso das teorias de saúde no cuidado a pacientes oncológicos, destacando os principais eixos na assistência de enfermagem oncológica.

\section{MÉTODO}

Trata-se de uma revisão integrativa da literatura cujo percurso metodológico teve início com a identificação do tema e da seguinte questão de pesquisa: quais teorias de saúde têm sido utilizadas para fundamentar os cuidados com pacientes portadores de neoplasias? A pergunta foi elaborada utilizando o acrônimo PICo (população; interesse; contexto), uma adaptação do PICO (população; intervenção; comparação; desfecho) amplamente utilizado em revisões que investigam a relação entre dois eventos ${ }^{(5)}$.

A busca ocorreu nos meses de julho e agosto de 2019, em pares, utilizando rede licenciada pela Universidade Federal do Maranhão, acessando as bases de dados: LILACS (Literatura Latino-Americana em Ciências da Saúde); MEDLINE (Medical Literature Analysis and Retrieval Sistem on-line) e PUBMED (U.S. National Library of Medicine).

Para busca nas bases de dados foram empregados os Descritores em Ciência da Saúde (DeCS): 1\# (Teoria de Enfermagem); 2\# (Neoplasia) nos idiomas português e inglês. Os cruzamentos foram realizados com o uso do operador booleano AND, da seguinte forma: 1\# AND 2\#.

Para a seleção dos artigos, foram adotados os seguintes critérios de inclusão: tratar de artigos publicados em português e inglês, com texto completo disponível nas bases de dados selecionadas e que tivessem relação com o problema de pesquisa. Foram excluídas cartas ao editor, resumos, opiniões de especialista, teses e dissertações. Optou-se por não realizar recorte temporal, de forma que todos os trabalhos disponíveis nas bases pesquisadas foram considerados.

Na primeira etapa, foram localizadas 932 publicações. Na segunda etapa, excluíram-se os artigos repetidos e os que não estavam disponíveis nos idiomas inglês e português e realizou-se a leitura dos títulos e resumos. $\mathrm{Na}$ 
terceira etapa, selecionaram-se os artigos que abordavam teorias de saúde na assistência a pacientes oncológicos para a leitura minuciosa. $\mathrm{Na}$ última fase da construção, foram selecionados os estudos que respondiam à questão de pesquisa, totalizando 16 artigos incluídos neste estudo (Figura 1).

Figura 1 - Fluxograma de construção do corpus da pesquisa. São Luís, Maranhão, agosto de 2019.

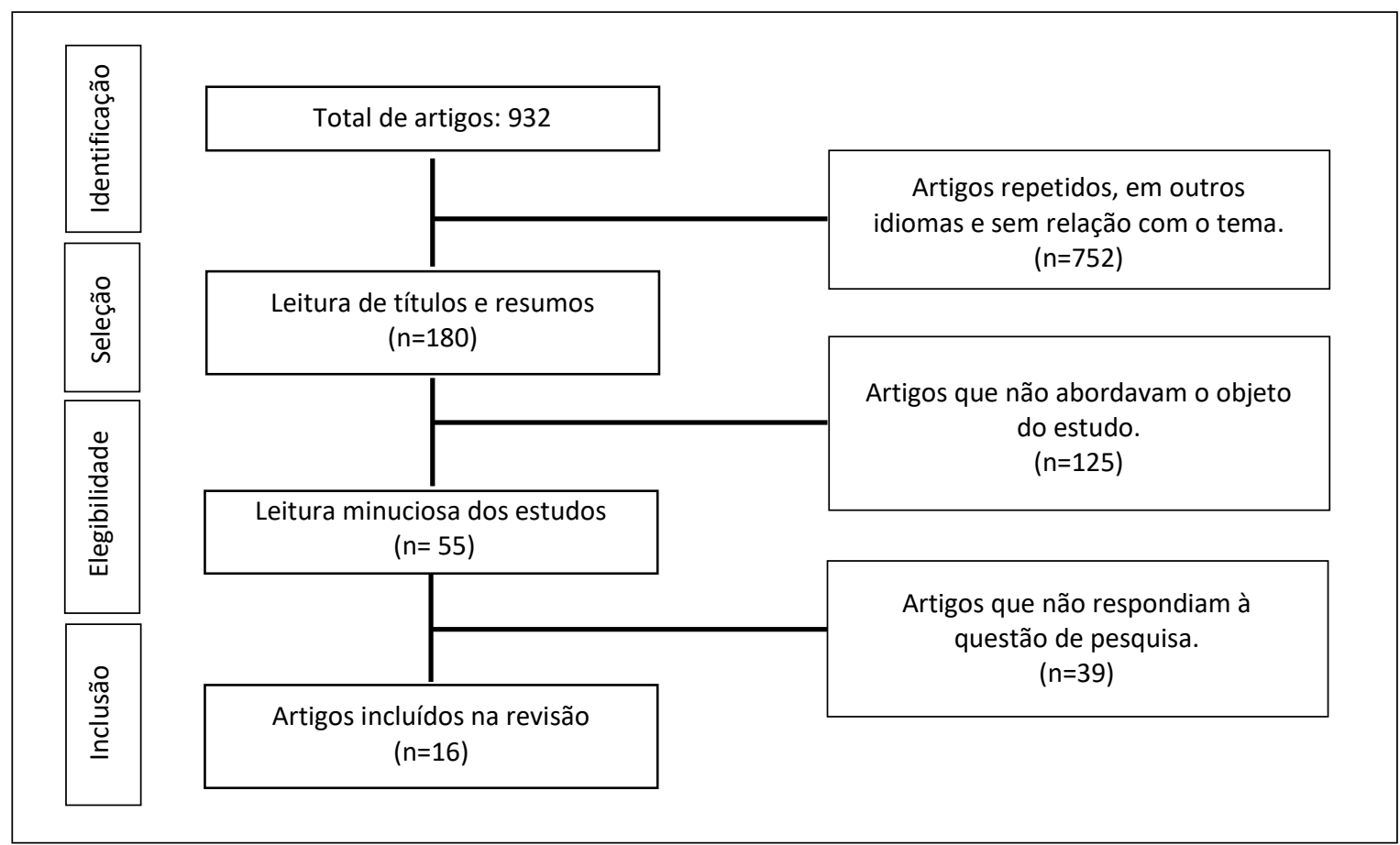

Fonte: compilado de informações dos autores.

Para a análise e extração dos dados, foi elaborado um roteiro com os seguintes dados: título do artigo, local e ano de realização, tipo de estudo, nível de evidência, teoria estudada e resultados.

Adotou-se uma hierarquia das evidências, segundo o delineamento da pesquisa, em cinco níveis: Nível 1 (evidências resultantes da metaanálise de múltiplos estudos clínicos controlados e randomizados); Nível 2 (evidências obtidas em estudos individuais com delineamento experimental); Nível 3 (evidências de estudos quase-experimentais); Nível 4 (evidências de estudos descritivos ou com abordagem qualitativa); Nível 5 (evidências provenientes de relatos de caso ou de experiência) $)^{(6)}$.

\section{RESULTADOS E DISCUSSÃO}

Dos dezesseis artigos que compuseram a amostra desta revisão, sete foram realizados no
Brasil, sete nos Estados Unidos e dois na Colômbia. Foram localizados trabalhos publicado, desde 1992 até 2018, sendo seis deles foram publicados nos últimos cinco anos, evidenciando a relevância cientifica do tema e o crescente interesse dos pesquisadores nos últimos anos.

Todos os artigos selecionados são de autoria da Enfermagem, a maioria apresentou abordagem teórica-reflexiva de revisão bibliográfica (9 artigos), 3 apresentaram abordagem quantitativa e 4 qualitativa, este, por meio de entrevista semiestruturada. É necessário considerar que estudos com essas abordagens são utilizados quando há conhecimento limitado sobre determinado fenômeno.

No Quadro 1, apresenta-se o título do artigo, local e ano de publicação, tipo de estudo, nível de evidência, teoria estudada e resultados. 
Quadro 1 - Caracterização das pesquisas sobre teorias de saúde utilizadas para fundamentar os cuidados com pacientes portadores de neoplasias, localizadas nas bases de dados LILACS, MEDLINE e PUBMED. São Luís, Maranhão, agosto de 2019.

\begin{tabular}{|c|c|c|c|c|c|}
\hline Título & $\begin{array}{l}\text { Local e ano } \\
\text { do estudo }\end{array}$ & Tipo de estudo & $\begin{array}{l}\text { Nível de } \\
\text { evidência }\end{array}$ & $\begin{array}{c}\text { Teoria } \\
\text { estudada }\end{array}$ & Resultados \\
\hline $\begin{array}{l}\text { Teoria dos sintomas } \\
\text { desagradáveis: } \\
\text { subsídios para o } \\
\text { manejo de sintomas } \\
\text { em crianças e } \\
\text { adolescentes } \\
\text { oncológicos }\end{array}$ & Brasil, 2015 & Teórico-reflexivo & Nível 4 & $\begin{array}{c}\text { Teoria de } \\
\text { Sintomas } \\
\text { Desagradáveis } \\
\text { (TSD) }\end{array}$ & $\begin{array}{c}\text { A teoria enfatiza a } \\
\text { complexidade e a interação } \\
\text { dos sintomas, as inter- } \\
\text { relações entre os mesmos, } \\
\text { os fatores que os } \\
\text { influenciam, e os resultados } \\
\text { e consequências destes (21). }\end{array}$ \\
\hline $\begin{array}{l}\text { Importância da } \\
\text { comunicação nos } \\
\text { cuidados paliativos } \\
\text { em oncologia } \\
\text { pediátrica: enfoque } \\
\text { na Teoria } \\
\text { Humanística de } \\
\text { Enfermagem }\end{array}$ & Brasil, 2013 & $\begin{array}{l}\text { Pesquisa de } \\
\text { campo, com } \\
\text { abordagem } \\
\text { qualitativa }\end{array}$ & Nível 5 & $\begin{array}{c}\text { Teoria } \\
\text { Humanística de } \\
\text { Enfermagem }\end{array}$ & $\begin{array}{c}\text { Surgiram duas categorias: } \\
\text { "estratégia para humanizar } \\
\text { o cuidar em enfermagem, } \\
\text { com ênfase no alívio do } \\
\text { sofrimento da criança" e } \\
\text { "estratégia para fortalecer } \\
\text { o vínculo de confiança } \\
\text { entre enfermeiro e criança" } \\
\text { (24). }\end{array}$ \\
\hline $\begin{array}{l}\text { Teorías y modelos } \\
\text { relacionados con } \\
\text { calidad de vida en } \\
\text { cáncer y enfermería }\end{array}$ & $\begin{array}{l}\text { Colômbia, } \\
2005\end{array}$ & $\begin{array}{l}\text { Revisão } \\
\text { integrativa }\end{array}$ & Nível 4 & $\begin{array}{l}\text { Teoria do } \\
\text { Autocuidado, } \\
\text { Incerteza, } \\
\text { Estresse e } \\
\text { Enfrentamento } \\
\text { de crises. }\end{array}$ & $\begin{array}{l}\text { Através da relação das } \\
\text { teorias, destacaram-se } \\
\text { medidas para auxiliar na } \\
\text { terapêutica e porporcionar } \\
\text { qualidade de vida aos } \\
\text { pacientes. Foram elas: } \\
\text { Medidas de bem-estar; } \\
\text { Bem-estar social; Bem- } \\
\text { estar psicológico; Bem- } \\
\text { estar espiritual (9). }\end{array}$ \\
\hline $\begin{array}{l}\text { O processo de } \\
\text { enfermagem no } \\
\text { tratamento das } \\
\text { alterações do } \\
\text { Papanicolau à luz de } \\
\text { duas teorias } \\
\text { convergentes: um } \\
\text { exercício didático }\end{array}$ & Brasil, 2004 & Relato de caso & Nível 5 & $\begin{array}{l}\text { Teoria das } \\
\text { Representações } \\
\text { Sociais } \\
\text { Teoria de } \\
\text { Imogene King }\end{array}$ & $\begin{array}{c}\text { Aliando-se às teorias, } \\
\text { constatou-se uma } \\
\text { convergência possível entre } \\
\text { ambas principalmente nos } \\
\text { aspectos que envolvem a } \\
\text { interação humana com } \\
\text { seus valores, crenças e que } \\
\text { são manifestos por meio da } \\
\text { comunicação (14). }\end{array}$ \\
\hline $\begin{array}{l}\text { Theories, Models, and } \\
\text { Frameworks Related to } \\
\text { Sleep-Wake } \\
\text { Disturbances in the } \\
\text { Context of Cancer }\end{array}$ & $\begin{array}{c}\text { Estados } \\
\text { Unidos, } 2010\end{array}$ & Revisão integrativa & Nível 4 & $\begin{array}{c}\text { Teoria do } \\
\text { Autocuidado do } \\
\text { Orem } \\
\text { Teoria dos } \\
\text { Sintomas } \\
\text { Desagradáveis }\end{array}$ & $\begin{array}{l}\text { Distúrbios do sono-vigília } \\
\text { afetam negativamente a } \\
\text { qualidade de vida }{ }^{(10)} \text {. }\end{array}$ \\
\hline $\begin{array}{l}\text { Enhancing Self-Efficacy } \\
\text { for Optimized Patient } \\
\text { Outcomes through the } \\
\text { Theory of Symptom } \\
\text { Self-Management }\end{array}$ & $\begin{array}{c}\text { Estados } \\
\text { Unidos, } 2014\end{array}$ & Qualitativo & Nível 4 & $\begin{array}{l}\text { Teoria dos } \\
\text { Sintomas de } \\
\text { Autogestão }\end{array}$ & $\begin{array}{c}\text { Descrição de como a } \\
\text { enfermagem em oncologia } \\
\text { pode usar intervenções de } \\
\text { autogestão de sintomas de } \\
\text { autoeficácia percebida para } \\
\text { melhorar o status funcional e } \\
\text { a qualidade de vida de seus } \\
\text { pacientes (7). }\end{array}$ \\
\hline $\begin{array}{l}\text { Using self-regulation } \\
\text { theory to develop an } \\
\text { intervention for } \\
\text { cancer-related fatigue. }\end{array}$ & $\begin{array}{c}\text { Estados } \\
\text { Unidos, } 2002\end{array}$ & Qualitativo & Nível 5 & $\begin{array}{c}\text { Teoria da } \\
\text { autorregulação }\end{array}$ & $\begin{array}{l}\text { De acordo com a teoria de } \\
\text { autorre-gulação, o manejo } \\
\text { inadequado do sintoma de } \\
\text { fadiga pode levar a um maior } \\
\text { desconforto por fadiga entre } \\
\text { os pacientes com câncer (23). }\end{array}$ \\
\hline
\end{tabular}




\begin{tabular}{|c|c|c|c|c|c|}
\hline Título & $\begin{array}{l}\text { Local e ano } \\
\text { do estudo }\end{array}$ & Tipo de estudo & $\begin{array}{l}\text { Nível de } \\
\text { evidência }\end{array}$ & $\begin{array}{c}\text { Teoria } \\
\text { estudada }\end{array}$ & Resultados \\
\hline $\begin{array}{l}\text { Testing a theory of } \\
\text { decision-making } \\
\text { derived from King's } \\
\text { systems framework in } \\
\text { women eligible for a } \\
\text { cancer clinical trial }\end{array}$ & $\begin{array}{l}\text { Estados } \\
\text { Unidos, } \\
2002\end{array}$ & Qualitativo & Nível 2 & $\begin{array}{c}\text { Teoria de } \\
\text { Imogene King }\end{array}$ & $\begin{array}{l}\text { A teoria de King propôs que } \\
\text { os conceitos de incerteza, } \\
\text { função e apoio social se } \\
\text { relacionam com a saúde } \\
\text { emocional, que por sua vez } \\
\text { se relaciona com a decisão } \\
\text { de tratamento (17). }\end{array}$ \\
\hline $\begin{array}{l}\text { Testing a theory for } \\
\text { health-related quality } \\
\text { of life in cancer } \\
\text { patients: a structural } \\
\text { equation approach. }\end{array}$ & $\begin{array}{l}\text { Estados } \\
\text { Unidos, } \\
1999\end{array}$ & Descritivo & Nível 2 & $\begin{array}{l}\text { Teoria baseada } \\
\text { no Modelo de } \\
\text { Adaptação de } \\
\text { Roy de } \\
\text { qualidade de } \\
\text { vida }\end{array}$ & $\begin{array}{l}\text { Os modos de resposta são } \\
\text { fisiológicos, autoconceito, } \\
\text { interdependência e função } \\
\text { de função, e a partir dessas } \\
\text { classificações, foram } \\
\text { apresentadas } 3 \text { proposições } \\
\text { relacionadas aos tipos de } \\
\text { respostas que o indivíduo } \\
\text { apresenta no diagnóstico } \\
\text { de câncer. As análises não } \\
\text { suportaram a proposição } \\
\text { de que todos os } 4 \text { modos } \\
\text { de resposta estavam inter- } \\
\text { relacionados (8). }\end{array}$ \\
\hline $\begin{array}{l}\text { The relationship of } \\
\text { self-concept and self- } \\
\text { care in children with } \\
\text { cancer }\end{array}$ & $\begin{array}{l}\text { Estados } \\
\text { Unidos, } \\
1998\end{array}$ & Descritivo & Nível 2 & $\begin{array}{l}\text { Teoria do } \\
\text { Autocuidado do } \\
\text { Orem }\end{array}$ & $\begin{array}{l}\text { As crianças tiveram maior } \\
\text { escore de autoconceito, } \\
\text { elas realizavam mais } \\
\text { atividades de autocuidado } \\
\text { e recebiam mais cuidados } \\
\text { dependentes de suas mães. } \\
\text { Os escores de autoconceito } \\
\text { dos grupos que faziam a } \\
\text { terapia e os que não faziam } \\
\text { não foram } \\
\text { significativamente } \\
\text { diferentes }\end{array}$ \\
\hline $\begin{array}{l}\text { Studies exploring self- } \\
\text { care for the person } \\
\text { coping with cancer } \\
\text { treatment: a review }\end{array}$ & $\begin{array}{l}\text { Estados } \\
\text { Unidos, } \\
1992\end{array}$ & $\begin{array}{c}\text { Revisão } \\
\text { integrativa }\end{array}$ & Nível 4 & $\begin{array}{l}\text { Teoria do } \\
\text { Autocuidado do } \\
\text { Orem }\end{array}$ & $\begin{array}{c}\text { O manipular a ingestão de } \\
\text { alimentos e líquidos, sono, } \\
\text { exercício é explorado, além } \\
\text { do papel que o } \\
\text { autocuidado exerce no } \\
\text { controle de náuseas e } \\
\text { vômitos causados pela } \\
\text { quimioterapia (11). }\end{array}$ \\
\hline $\begin{array}{l}\text { Incertidumble frente } \\
\text { al diagnóstico de } \\
\text { câncer }\end{array}$ & $\begin{array}{l}\text { Colômbia, } \\
2018\end{array}$ & Qualitativo & Nível 2 & $\begin{array}{l}\text { Teoria da } \\
\text { Incerteza na } \\
\text { Doença de } \\
\text { Mishel }\end{array}$ & $\begin{array}{c}\text { A incerteza é um } \\
\text { sentimento que invade os } \\
\text { pacientes com doença } \\
\text { crônica. Temas emergiram- } \\
\text { apoio da equipe de saúde e } \\
\text { familiares no diagnóstico; } \\
\text { Atitude positiva; } \\
\text { Sofrimento em vista da } \\
\text { adversidade; Segurando em } \\
\text { Deus e a adversidade do } \\
\text { sistema de saúde (18). }\end{array}$ \\
\hline
\end{tabular}




\begin{tabular}{|c|c|c|c|c|c|}
\hline Título & $\begin{array}{l}\text { Local e ano do } \\
\text { estudo }\end{array}$ & Tipo de estudo & $\begin{array}{l}\text { Nível de } \\
\text { evidência }\end{array}$ & $\begin{array}{c}\text { Teoria } \\
\text { estudada }\end{array}$ & Resultados \\
\hline $\begin{array}{l}\text { Prevenção do câncer } \\
\text { de colo uterino de } \\
\text { quilombolas à luz da } \\
\text { Teoria de Leininger }\end{array}$ & Brasil, 2018 & Qualitativo & Nível 2 & $\begin{array}{l}\text { Teoria do } \\
\text { Cuidado } \\
\text { Transcultural } \\
\text { de Madeleine } \\
\text { Leininger }\end{array}$ & $\begin{array}{l}\text { As quilombolas apontaram } \\
\text { como práticas preventivas de } \\
\text { câncer do colo uterino o uso } \\
\text { de plantas medicinais, e o } \\
\text { cuidado profissional, } \\
\text { caracterizado pela realização } \\
\text { do Papanicolau. Contudo, } \\
\text { uma maioria de mulheres } \\
\text { não realizavam prevenção } \\
\text { (19). }\end{array}$ \\
\hline $\begin{array}{c}\text { Existential } \\
\text { experience of } \\
\text { children with cancer } \\
\text { under palliative care }\end{array}$ & Brasil, 2018 & Qualitativo & Nível 2 & $\begin{array}{c}\text { Teoria } \\
\text { Humanística } \\
\text { da } \\
\text { Enfermagem }\end{array}$ & $\begin{array}{l}\text { A teoria da enfermagem } \\
\text { humanística na oncologia } \\
\text { pediátrica pode aperfeiçoar e } \\
\text { orientar a prática de } \\
\text { enfermeiros, uma vez que } \\
\text { essa teoria se baseia no } \\
\text { encontro e no diálogo } \\
\text { autêntico entre quem cuida e } \\
\text { quem é cuidado (15). }\end{array}$ \\
\hline $\begin{array}{l}\text { Aconselhamento e } \\
\text { assistência espiritual } \\
\text { a pacientes em } \\
\text { quimioterapia: uma } \\
\text { reflexão à luz da } \\
\text { teoria de Jean } \\
\text { Watson }\end{array}$ & Brasil, 2018 & Teórico-reflexivo & Nível 4 & $\begin{array}{l}\text { Teoria do } \\
\text { Cuidado } \\
\text { Humano }\end{array}$ & $\begin{array}{l}\text { A análise crítica de métodos } \\
\text { de aconselhamento } \\
\text { psicológico, teológico e } \\
\text { psicoterápico indica } \\
\text { aproximações com a Teoria } \\
\text { do Cuidado Humano, } \\
\text { promovendo o } \\
\text { aprimoramento de } \\
\text { intervenções espirituais } \\
\text { direcionadas a pacientes em } \\
\text { quimioterapia (20). }\end{array}$ \\
\hline $\begin{array}{c}\text { Experiência } \\
\text { existencial de } \\
\text { crianças em } \\
\text { tratamento } \\
\text { quimioterápico sobre } \\
\text { a importância do } \\
\text { brincar }\end{array}$ & Brasil, 2018 & Qualitativa & Nível 5 & $\begin{array}{c}\text { Teoria } \\
\text { Humanística } \\
\text { da } \\
\text { Enfermagem }\end{array}$ & $\begin{array}{l}\text { As crianças compreendem } \\
\text { sobre sua doença e seu } \\
\text { tratamento, evidenciando as } \\
\text { privações vivenciadas por } \\
\text { elas e a situação de } \\
\text { desequilíbrio emocional. } \\
\text { Brincar, suscitou sentimentos } \\
\text { positivos, constituindo uma } \\
\text { maneira de o tempo passar } \\
\text { mais rápido (22). }\end{array}$ \\
\hline
\end{tabular}

Fonte: compilado de informações dos autores.

Com base nos materiais analisados, foi possível apontar 4 eixos principais na assistência de enfermagem oncológica que serão discutidos a seguir.

\section{Câncer, enfermagem e cuidado fundamentado em teorias}

Historicamente as primeiras iniciativas desenvolvidas para o controle do câncer ainda são recentes quando comparadas a outras patologias, tendo sido orientadas, quase exclusivamente, para o diagnóstico e tratamento da doença.Com isso, as ações de prevenção e informação da população ficaram prejudicadas, sendo justificadas pela própria incipiência do conhecimento da etiologia do câncer ${ }^{(1)}$.

O que permite compreender como o câncer tornou-se uma doença temida por todos, envolvida em mitos e crenças que surgiram da falta de conhecimento e informação por parte da maioria da população(1).

Mesmo hoje em dia, quando a medicina eleva o câncer à condição de doença crônica, em razão da alta tecnologia de diagnóstico, muitas pessoas têm a condição de identificação e início de tratamento precoce, proporcionando cura e aumento da sobrevida. Porém, a realidade se depara com o aumento do número de casos da 
doença, o que muito tem preocupado e chamado a atenção dos gestores de políticas públicas em saúde, dos profissionais de saúde e da comunidade $^{(1)}$.

Tal situação é de grande relevância para as profissões da área de saúde e, especialmente, para a enfermagem, considerando a importância da visão dos doentes acerca da doença e do tratamento para melhor gerenciamento de suas condições de saúde, o que favorece maior participação da pessoa no controle do tratamento e do atendimento das suas necessidades para melhorar sua qualidade de vida ${ }^{(7)}$.

Para fundamentar, cientificamente, os cuidados realizados temos as teorias de enfermagem, que estão dominantes na literatura nos últimos 40 anos, contribuindo para o seu desenvolvimento como profissão. A era da teoria, em conjunto com a consciência da enfermagem como profissão e disciplina acadêmica, emergiu dos debates e discussões dos anos 60. A transição de vocação para profissão, nos anos 70 , foi decisiva para a enfermagem, por questionar qual a disciplina em que deveria se basear, sendo que a resposta foi na ciência de enfermagem ${ }^{(4)}$.

\section{O autocuidado e o paciente oncológico}

O cuidado de enfermagem ao paciente oncológico abrange todos os grupos etários e, em geral, é realizado em diversificados ambientes como hospitais, centros de reabilitação, residências e comunidades. Logo, esse cuidado tão específico deve contemplar a realidade de vida do paciente, pois a experiência da doença é única para cada indivíduo e cada um irá expressar necessidades coletivas que são expressões de determinantes sociais, econômicos e culturais ${ }^{(2-8)}$.

Há tempos que o cuidado vem sendo estudado e refletido pela enfermagem. Porém, ainda estamos longe de uma definição clara e objetiva. Essa dificuldade de definição pode estar relacionada à complexidade do cuidado, uma vez que emerge das relações interpessoais entre seres humanos carregados de diferentes crenças, valores e necessidades ${ }^{(2)}$. Dos 16 estudos analisados neste trabalho, 4 levantaram a Teoria do autocuidado de Orem para a melhoria da assistência de enfermagem aos pacientes.

Ao formular sua teoria, Dorothea Orem, por meio desta, afirma que o sujeito adulto só exige assistência de enfermagem quando existe a incapacidade de manter, frequentemente, a quantidade e a qualidade do autocuidado ${ }^{(9)}$. Ou seja, o sujeito tem ausência de capacidade para sozinho manter a sustentação da vida, da saúde, recuperar-se da doença ou lesão, assim como enfrentar os seus efeitos. ${ }^{(9-10)}$.

O autocuidado consiste na prática de atividades que as pessoas iniciam e conduzem, em certos períodos de tempo, por seus próprios meios e no interesse de manter uma operação saudável e viva, e continuar com desenvolvimento pessoal e bem-estar ${ }^{(9)}$. Já o déficit de autocuidado descrito por Orem ocorre quando a relação entre as propriedades humanas de necessidade terapêutica e a capacidade de autocuidado desenvolvida não são operativas ou adequado para conhecer e cobrir alguns ou todos os componentes da necessidade terapêutica de autocuidado existente ${ }^{(10)}$.

A Teoria do Autocuidado de Dorothea Orem, foi desenvolvida a partir de um marco conceitual no qual a teórica acredita que o profissional de enfermagem juntamente com o cliente, deva identificar déficits de capacidade no atendimento das necessidades individuais de autocuidado, procurando desenvolver, nesses indivíduos os potenciais já existentes para a prática do autocuidado ${ }^{(10)}$.

Dessa forma, o profissional de Enfermagem funciona no autocuidado como regulador do sistema. Ele identifica os déficits de competência em relação à demanda de autocuidado, faz pelo indivíduo aquilo que ele não pode fazer, ensina, orienta e promove o desenvolvimento das capacidades do indivíduo para que ele possa se tornar independente da assistência de enfermagem assumindo seu autocuidado ${ }^{(11)}$.

Em uma pesquisa realizada no Rio de Janeiro-Brasil, com pacientes oncológicos estomizados identificou-se que esses pacientes ainda precisam tomar consciência para entender os efeitos e resultados de sua condição patológica, modificando seu autoconceito, adaptando formas específicas de cuidado de saúde, para aprender a viver com os efeitos da intervenção médica e a desenvolver um estilo de vida que promova um continuado desenvolvimento pessoal ${ }^{(12)}$.

Em um estudo, ao desenvolveram uma pesquisa com 74 crianças com câncer e suas mães. Os resultados mostraram, pequenas, mas significativas correlações entre as variáveis, fornecendo algumas evidências de que,se as crianças tivessem maior escore de autoconceito, elas realizariam mais atividades de autocuidado e recebiam mais cuidados dependentes de suas mães. Este estudo, apóia a teoria de Orem e 
reforça a necessidade de promover o autoconceito positivo ${ }^{(13)}$.

Percebe-se que o entendimento sobre a patologia faz com que os indivíduos fortaleçam o autoconceito e consigam atingir o autocuidado de forma satisfátoria, sempre tendo como base fundamentadora o cuidado e acompanhamento de enfermagem este, por sua vez, embasado na teoria do autocuidado de Dorothea Orem ${ }^{(11)}$.

\section{Processo de ação, reação e interação na enfermagem}

Em um estudo realizado em 2005, mostra como o referencial teórico de Imogene King pode definir o processo de enfermagem como um processo de ação, reação e interação, pelo qual o enfermeiro e cliente compartilham informações sobre suas percepções na situação de enfermagem. Pode-se definir ainda a saúde como um estado dinâmico no ciclo de vida de um ser humano que implica em ajustamentos contínuos a estressores, no ambiente interno e externo, por meio do uso de recursos próprios para alcançar o máximo potencial para a vida diária ${ }^{(14)}$.

Assim, os conceitos fazem parte da estrutura conceitual da teoria acima citada a qual é composta por três sistemas considerados abertos: o pessoal, o interpessoal e o social e, em cada um, há um grupo de conceitos relevantes. $\mathrm{Na}$ compreensão de King a comunicação é o elemento que somado à percepção promove a interação entre as pessoas, a comunicação como um é instrumento para aperfeiçoar a prática assistencial, e conseguir gerenciar as implicações do processo de paliação infantil(15-16).

A teoria derivada da estrutura de King propôs que os conceitos de incerteza, função e apoio social se relacionam com a saúde emocional (esperança e estado de humor) que, por sua vez, se relaciona com a decisão de tratamento. Um desenho de estudo correlacional foi usado para testar a teoria em uma amostra de 40 mulheres. As descobertas forneceram evidências empíricas da adequação da estrutura de King e apoiaram, em parte, as relações teorizadas entre os fatores críticos $^{(17-18)}$.

A incorporação na prática assistencial dos conceitos propostos por Imogene King permitiu compreender que cuidar, tendo como meta a saúde e, consequentemente, a melhoria da qualidade de vida do cliente, assume um significado transcedente e que, ser cuidado, certamente contribui para amenizar o sofrimento presente na experiência de estar vivendo os limites entre a vida e morte ${ }^{(16)}$.

\section{Outras formas de cuidar}

Partindo desse pressuposto, notamos que o cuidar em enfermagem é amplo e deve ter por base um conhecimento científico, norteando suas ações e abrindo caminhos para o pensamento crítico do profissional enfermeiro.

É necessário que o enfermeiro enxergue o paciente de forma holística e como um sujeito que possui crenças, valores e culturas próprias e que devem ser incorporadas durante o cuidado, para que assim se estabeleça uma relação de confiança que facilite a assistência prestada e um cuidado de saúde culturalmente competentes. Assim, acredita-se que conhecer e compreender a cultura de populações, bem como a relação destas com os comportamentos de saúde e as práticas de cuidados é fundamental para a assistência de profissionais de saúde ${ }^{(19)}$.

Entendendo o paciente como um indivíduo com crenças, parte dos problemas enfrentados por pacientes, no ambulatório de quimioterapia, pode ser minimizada por uma assistência espiritual, cabe ao enfermeiro perceber essa necessidade, muitas vezes oculta nas queixas e sintomas do cliente. Ainda que o enfermeiro não seja um praticante religioso, precisa estar presente e perceber quando a causa de sofrimento do cliente se associa a sentimentos de punição divina. A assistência espiritual deve ser realizada para dirimir o confronto entre crenças religiosas e cuidados de saúde ${ }^{(20)}$.

O cuidado a crianças e adolescentes oncológicos, mostra que esses clientes experimentam uma variedade de sintomas, os quais, por vezes, ocorrem simultaneamente. Esses sintomas estão associados a piores prognósticos, incluindo baixa sobrevida, redução da adesão ao tratamento e uma qualidade de vida diminuída ${ }^{(21)}$. A brinquedoterapia mostrou-se eficaz, numa melhor adesão ao tratamento, pois suscitava sentimentos positivos, permitindo uma experiência menos desagradável I(22).

A importância de se avaliar e intervir sob tais sintomas, não é só em termos de sobrevida do paciente, mas também de qualidade de vida, durante e após o tratamento, é atualmente parte integrante dos pilares da pesquisa em oncologia clínica e prioridade em pesquisas na enfermagem

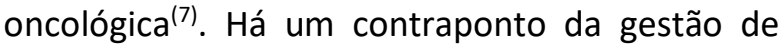
sintomas, onde o manejo inadequado de um ou 
mais sintomas trazem maiores consequências a um paciente já debilitado pela doença de base e, portanto, a teoria da autorregulação dos sintomas deve se fazer presente no planejamento da assistência de enfermagem oncológica antes mesmo do primeiro contato com o tratamento ${ }^{(23)}$.

No campo da enfermagem utilizamos algumas ferramentas fundamentais para o desenvolvimento do cuidado. A comunicação é uma delas, importante para a prática dos cuidados com todos os tipos de pacientes, inclusive aqueles sem possibilidades terapêuticas de cura, especialmente quando se trata de criança com câncer, a comunicação é uma estratégia para a criação de vínculo e confiança da criança com o enfermeiro, tornando de suma importância para a promoção dos cuidados paliativos ${ }^{(24)}$.

Nesse contexto, quando uma criança adoece de câncer, ocorrem mudanças de hábitos, restrições, isolamento, afastamento das atividades de rotina, as internações recorrentes, que causam intenso sofrimento. A comunicação eficaz é considerada instrumento fundamental para o cuidado integral e humanizado porque, por meio dela, é possível reconhecer e acolher, empaticamente, as necessidades do paciente ${ }^{(25)}$.

\section{CONSIDERAÇÕES FINAIS}

A análise do uso das teorias de saúde, no cuidado a pacientes oncológicos, oportunizou a identificação de eixos relevantes na assistência de enfermagem oncológica, alcançando, dessa forma, o objetivo deste estudo. Há potencial para o avanço da ciência no campo da enfermagem, oncologia e teorias científicas, bem como suas interações. Do mesmo modo, a avaliação de mudanças na gravidade dos sintomas, ao longo do tempo e do cuidar é uma proposta promissora.

O cuidar, em enfermagem, é um processo de relação mútua entre seres humanos. Nessa relação, o cuidado, por meio de ferramentas apresenta-se como eixo para o seu desenvolvimento, porquanto é concebida pelos próprios enfermeiros como um dos instrumentos mais relevantes.

Percebeu-se que há necessidade em se expandir os conhecimentos e pesquisas, utilizando-se como bases as teorias científicas e o paciente oncológico, cliente este que é alvo de inúmeras complicações, durante seu tratamento e, até mesmo, após a cura. O conhecimento teórico científico mostra artifícios para o cuidar de forma concreta.

\section{REFERÊNCIAS}

1- Ministério da Saúde (BR). Ações de Enfermagem para o controle do câncer: Uma proposta de integração. 3a ed rev, atual e ampl. Rio de Janeiro: INCA; 2008.

2- Cardoso JA, Santos MNP, Morgado SSM. Atuação do enfermeiro no cuidado ao paciente oncológico no domicílio. Rev Eletrôn Atualiza Saúde 2017 [citado em 10 abr 2018]; 6(6):36-42. Available in: http://atualiza revista.com.br/wpcontent/uploads/2017/08/Atuação-doenfermeiro-no-cuidado-do-paciente-oncológicono-domicílio-v-6-n-6.pdf

3- Merino MFGL, Silva PLAR, Carvalho MDB, Pelloso SM, Bladissera VDA, Higarashi IH. Teorias de enfermagem na formação e na prática profissional: Percepção de pós-graduandos de enfermagem. Rev RENE 2018; 19:e3363. DOI: 10.15253/2175-67832018193363

4- Moreira LHD, Silva RG. Identificação das principais teorias de enfermagem utilizadas nos diferentes níveis de atenção à saúde do município de Assis-SP. In: Anais do 170 Congresso de Iniciação Científica, 2017; São Paulo. São Paulo: SEMESP; 2017. p. 1-10.

5- Galvão TF, Pereira MG. Revisões sistemáticas da literatura: passos para sua elaboração. Epidemiol Serv Saúde 2014;23(1): 183-4. DOI: 10.5123/S1679-49742014000100018

6- Souza MT, Silva MD, Carvalho R. Integrative review: What is it? How to do it? Einstein 2010; 8(1):102-6. DOI: 10.1590/s1679-45082010rw1134 7- Hoffman AJ. Enhancing self-efficacy for optimized patient outcomes through the theory of symptom self-management. Cancer Nurs. 2013;36(1):E16-E26.

DOI: 10.1097/NCC.0b013e31824a730a

8- Nuamah IF, Cooley ME, Fawcett J, Mccorkle R. Testing a theory for health-related quality of life in cancer patients: A structural equation approach. Res Nurs Health 1999;25(1):74-80. DOI: 10.1002/(sici)1098-240x(199906)22:3<231::aidnur5>3.0.co;2-6

9- Triviño ZG, Sanhueza O. Teorías y modelos relacionados con calidad de vida en cáncer y enfermería. Aquichan 2005 [citado em11 abr 2018]; 5(1):20-31. Available in: http://www.scielo.org.co/scielo.php? script=sci_arttext\&pid=S1657-

59972005000100003

10- Julie LO, Janet SC. Theories, models, and frameworks related to sleep-wake disturbances in the context of cancer. Cancer Nurs 2009;32(2):90106. DOI: $10.1097 / 01 . N C C .0000339261 .06442 .7 d$ 
11- Studies exploring self-care for the person coping with cancer treatment: A review. Int J Nurs Stud. 1992; 29(2):191-204. DOI: 10.1016/00207489(92)90008-5

12- Silva DF. O desafio do autocuidado de pacientes oncológicos estomizados: Da reflexão à ação [dissertação]. Niterói (RJ):Universidade Federal Fluminense; 2013.

13- Mosher RB, Moore JB. The relationship of selfconcept and self-care in children with cancer. Nurs Sci Q. 1998;11(3):116-22. DOI: 10.1177/089431849801100310

14- Mariotti SR, Labronici LM, Mantovani MF. O processo de enfermagem no tratamento das alterações do Papanicolau à luz de duas teorias convergentes: UM exercício didático. Cogitare Enferm. 2005;10(1):66-70. DOI: 10.5380/ce.v10i1.4675

15- França JRF, Costa SFG, Lopes MEL, Nóbrega MML, Batista PSS, Oliveira RC. Existential experience of children with cancer under palliative care. Rev Bras Enferm. 2018;71(3):1320-7. DOI: 10.1590/0034-7167-2016-0493

16- Miranda SPL. Diagnósticos de enfermagem em clientes oncológicos críticos em cuidados paliativos fundamentados na teoria do alcance de metas de King [dissertação]. Brasília (DF): Universidade de Brasília; 2015.

17- Ehrenberge RHE, Alligood MR, Thomas SP, Wallace DC, Licavoli CM. Testing a theory of decision-making derived from King's systems framework in women eligible for a cancer clinical trial. Nurs Sci Q. 2002;15(2):156-63. DOI: 10.1177/08943180222108822

18- Ramírez-Perdomo CA, Rodríguez-Vélez ME, Perdomo-Romero AY. Incertidumble frente al diagnóstico de câncer. Texto Contexto Enferm. 2018;27(4):e5040017. DOI: 10.1590/0104$\underline{07072018005040017}$

19- Fernandes ETBS, Nascimento ER, Ferreira SL, Coelho EAC, Silva LR, Pereira COJ. Prevenção do câncer de colo uterino de quilombolas à luz da Teoria de Leininger. Rev Gaúcha Enferm. 2018;39:e2016-0004. DOI: 10.1590/19831447.2018.2016-0004

20- Mendonça AB, Pereira ER, Barreto BMF, Silva RMCRA. Aconselhamento e assistência espiritual a pacientes em quimioterapia: Uma reflexão à luz da teoria de Jean Watson. Esc Anna Nery Rev. 2018;22(4):e20180081. DOI: 10.1590/2177-9465ean-2018-0081

21- Lopes-Junior LC, Bomfim EO, Nascimento LC, Silva GP, Lima RAG. Teoria dos sintomas desagradáveis: subsídios para o manejo de sintomas em crianças e adolescentes oncológicos. Rev Gaúcha Enferm. 2015;36(3):109-12. DOI: 10.1590/1983-1447.2015.03.51465

22- Morais GSN, Costa SFG, França JRFS, Duarte MCS, Lopes MEL, Batista PSS. Experiência existencial de crianças em tratamento quimioterápico sobre a importância do brincar. Rev RENE 2018;19:e3359. DOI: 10.15253/21756783.2018193359

23- Reuille KM. Using self-regulation theory to develop an intervention for cancer-related fatigue. Clin Nurse Spec. 2002;16(6):312-9. DOI: 10.1097/00002800-200211000-00015

24- França JRFS, Costa SFG, Lopes MEL, Nóbrega MML, França ISX. Importância da comunicação nos cuidados paliativos em oncologia pediátrica: enfoque na Teoria Humanística de Enfermagem. Rev Latino-Am Enfermagem 2013;21(3):1-7. DOI: 10.1590/S0104-11692013000300018

25- Chico CE, Nascimento EC, Castanheira L, Lima RAG. Children and adolescents with cancer: Experiences with chemotherapy. Rev Latino-Am Enfermagem 2010;18(5):864-72. DOI: 10.1590/S0104-11692010000500005

NOTA: Esse artigo não faz parte de relatórios de pesquisa, tese, dissertação ou monografia de final de curso.

Received in: 03/02/2020

Approved in: 01/06/2020

Mailing address:

Thalita dos Santos Costa

Avenida dos Portugueses, 1966, Vila Bacanga, São Luís - MA, Brazil, CEP: 65080-030.

E-mail: thalitasc@live.com 